\title{
Microangiopatías trombóticas primarias: una revisión narrativa
}

\section{Primary thrombotic microangiopathies: a narrative review}

\author{
Natalia Henao-Piedrahita ${ }^{1}$
}

\begin{abstract}
Resumen. La microangiopatía trombótica (MAT) es un síndrome donde hay formación de microtrombos en la circulación que llevan a anemia hemolítica microangiopática (AHMA) y trombocitopenia con falla multiorgánica, debido a la isquemia de los tejidos. Las MAT pueden ser primarias sin causa subyacente asociada, como la púrpura trombocitopénica trombótica debida a deficiencia de la enzima ADAMTS13, el síndrome hemolítico urémico debido a la toxina Shiga de Escherichia coli enterohemorrágica, y la MAT producida por alteraciones en la regulación del complemento. Adicionalmente, pueden ser secundarias a enfermedades malignas, infecciosas, metabólicas, autoinmunes o inducidas por el embarazo. Estas patologías requieren diagnóstico y tratamiento oportunos debido a que tienen alta morbimortalidad y se asocian a complicaciones que incluyen enfermedad renal, alteraciones neurológicas como convulsiones, accidente cerebrovascular, coma y muerte. El tratamiento es multidisciplinario y se enfoca en el soporte hemodinámico, transfusional y en el manejo de la etiología cuando esta es identificada. La siguiente revisión pretende explicar de forma clara y precisa los aspectos generales de las MAT primarias.
\end{abstract}

Palabras clave: microangiopatías trombóticas, púrpura trombocitopénica trombótica, síndrome hemolítico urémico, síndrome hemolítico urémico atípico, anemia hemolítica, trombocitopenia.

Abstract. Thrombotic microangiopathy (TMA) is a syndrome characterized by the formation of microthrombi in the circulation leading to microangiopathic hemolytic anemia (MAHA) and thrombocytopenia, with multiorgan failure due to tissue ischemia. TMA can be primary with no associated underlying cause, such as thrombotic thrombocytopenic purpura due to ADAMTS13 deficiency, hemolytic uremic syndrome due to the Shiga toxin from enterohemorrhagic Escherichia coli, or due to complement dysregulation. Furthermore, TMA can be secondary to malignant,

\footnotetext{
${ }^{1}$ Médica General, Fundación Universitaria San Martín. Sabaneta, Colombia. E-mail: natalia.henao.pi@gmail.com.

Conflicto de interés: la autora declara que no tiene conflicto de interés.

Medicina \& Laboratorio 2021;25:485-499. https://doi.org/10.36384/01232576.432.

Recibido el 27 de octubre de 2020; aceptado el 23 de febrero de 2021. Editora Médica Colombiana S.A., $2021^{\circ}$.
} 
infectious, metabolic or autoimmune diseases, or induced by pregnancy. These conditions require a timely diagnosis and treatment due to their associated high morbidity and mortality, and complications like renal disease, neurological disorders such as seizures, stroke, coma and death. Treatment is multidisciplinary and focuses on hemodynamic and transfusion support, and on the management of the etiology when it is identified (daily plasma exchange, eculizumab or management of underlying disease). This review aims to discuss the general aspects of primary thrombotic microangiopathies.

Keywords: thrombotic microangiopathies, thrombotic thrombocytopenic purpura, hemolytic uremic syndrome, atypical hemolytic uremic syndrome, hemolytic anemia, thrombocytopenia.

\section{Introducción}

El primer síndrome de microangiopatía trombótica (MAT) fue descrito por primera vez en 1925 por Eli Moschcowitz, quien atribuyó las manifestaciones clínicas de debilidad, palidez, fiebre y petequias de una joven de 16 años a "un potente veneno con efectos aglutinantes y hemolíticos"; posteriormente, la paciente desarrolló hemiparesia, coma y falleció. La autopsia reportó la presencia de trombos hialinos en la microcirculación del corazón, riñón, bazo e hígado [1].

Las MAT son un conjunto de procesos que cursan con una alteración del endotelio vascular y formación de microtrombos, que presentan hallazgos característicos como anemia hemolítica microangiopática con esquistocitos en el extendido de sangre periférica y trombocitopenia de intensidad variable [2]. Pueden ser hereditarias o adquiridas, y se clasifican en primarias y secundarias. Las MAT primarias son aquellas que ocurren de manera espontánea sin una causa subyacente asociada; por ejemplo, la púrpura trombocitopénica trombótica (PTT) debida a deficiencia de ADAMTS13 (del inglés, A Disintegrin and Metalloproteinase with a Thrombospondin Type 1 Motif, Member 13), el síndrome hemolítico urémico (SHU) asociado a toxina Shiga de Escherichia coli enterohemorrágica (también denominado SHU típico) y la MAT mediada por una alteración en la regulación del complemento (también denominado SHU atípico) $[3,4]$. En estas condiciones hay un defecto heredado o adquirido que promueve cambios a nivel vascular, como edema de células endoteliales y del espacio subendotelial, engrosamiento de la pared del vaso y microtrombos de plaquetas en arteriolas pequeñas y capilares [5]. Por su parte, las MAT secundarias se dan en el contexto de enfermedades sistémicas subyacentes y están asociadas a enfermedades neoplásicas, infecciosas, metabólicas, autoinmunes y gestacionales, entre otras $[3,6,7]$. Su diagnóstico se realiza por biopsia de tejidos; sin embargo, se puede inferir su diagnóstico con la observación de anemia hemolítica microangiopática (AHMA), trombocitopenia y Coombs negativo en un escenario clínico adecuado. El tratamiento de esta entidad se enfoca en el manejo de las condiciones desencadenantes, soporte hemodinámico, transfusional y tratamiento de la causa específica cuando es identificada. El objetivo de este artículo es 
explicar de forma clara y precisa aspectos generales como la fisiopatología, manifestaciones clínicas, diagnóstico y tratamiento de las tres microangiopatías trombóticas primarias.

\section{Generalidades}

Los síndromes de microangiopatías trombóticas comparten algunas características clínicas, pero cada una de ellas tiene una fisiopatología y manejo terapéutico distintos. Las MAT son un grupo de patologías que afectan el endotelio vascular y se caracterizan por la presencia de anemia hemolítica microangiopática no inmune y trombocitopenia, que son producidas por la formación de trombos de plaquetas y la fragmentación mecánica de los glóbulos rojos a su paso por la microcirculación. Los pacientes presentan hallazgos característicos de hemólisis como la disminución de la hemoglobina y haptoglobina, y aumento de la deshidrogenasa láctica, reticulocitos y bilirrubina indirecta, asociados a la presencia de esquistocitos en el extendido de sangre periférica.

Los sitios típicos de compromiso son el riñón y el sistema nervioso central. La lesión renal aguda severa es un hallazgo prominente de las MAT, excepto en la PTT, en la cual es rara, y los pacientes pueden presentar manifestaciones neurológicas leves como cefalea y confusión, o severas con convulsiones, enfermedad cerebrovascular, coma y/o muerte [8]. Otros órganos también pueden estar comprometidos, como el corazón, el tracto gastrointestinal y la piel, ya que pueden manifestarse hallazgos clínicos como síndrome coronario agudo, dolor abdominal, disentería o lesiones purpúricas en piel. También, se han encontrado trombos microvasculares en los pulmones, pero rara vez este hallazgo se hace clínicamente evidente [9]. La autopsia de los pacientes con MAT generalmente muestra trombos microvasculares en la mayoría de órganos [5]. Algunas enfermedades que pueden tener características similares a las MAT se enuncian en la tabla 1.

\section{MAT primarias}

Las MAT primarias pueden ser hereditarias $\mathrm{O}$ adquiridas, y se presentan de manera espontánea, sin una causa subyacente aparente. Las principales incluyen la PTT debida a deficiencia de ADAMTS13, el SHU asociado a toxina Shiga de Escherichia coli enterohemorrágica y la MAT mediada por complemento (también denominado SHU atípico), que se describen a continuación $[3,4,10,11]$.

\section{Púrpura trombocitopénica trombótica (PTT)}

Es un desorden oclusivo microcirculatorio que se caracteriza por la aglomeración plaquetaria sistémica e isquemia de órganos, asociados a AHMA y trombocitopenia severa [12]. Es una MAT rara que amenaza la vida y es definida por una deficiencia severa de ADAMTS13 (típicamente con una actividad inferior al 10\%) $[5,13]$. Puede tener origen congénito (síndrome de Upshaw-Shulman) o puede ser adquirida debido a la producción de anticuerpos contra la enzima ADAMTS13 [14].

\section{Epidemiología}

La PTT congénita o hereditaria es rara y representa menos del $5 \%$ de todas las PTT, mientras que la incidencia de PTT adquirida es de 3 casos por millón de adultos por año, de acuerdo con los da- 
Tabla 1. Diagnósticos diferenciales de las microangiopatías trombóticas

\begin{tabular}{ll}
\hline & Bacterias gram positivas, Rickettsiae, Borrelia, \\
& Brucella, Chlamydia, Clostridium difficile, \\
& Ehrlichia, Legionella, Leptospira, Mycobacteria, \\
citomegalovirus, virus de la hepatitis, VIH, & influenza A, parvovirus B19, varicella zoster, \\
Enfermedades infecciosas & HHV-6, EBV, dengue, Coxsackie virus, norovirus, \\
& Aspergillus, Blastomyces, Candida albicans, \\
& Cryptococcus neoformans, Babesia, malaria, \\
& endocarditis infecciosa, sepsis \\
Enfermedades autoinmunes & Lupus eritematoso sistémico, síndrome \\
antifosfolípido, esclerodermia (esclerosis & sistémica), vasculitis sistémica, síndrome de \\
& Evans, enfermedad de Graves
\end{tabular}

Enfermedades malignas

Síndrome mielodisplásico, leucemia aguda, tumores de pulmón, mama, próstata, páncreas o gastrointestinales

Enfermedades relacionadas con el embarazo

Preeclampsia, eclampsia, síndrome de HELLP

Trasplante

Órgano sólido y de células hematopoyéticas

Síndrome de Wiskott-Aldrich, síndrome MYH9

Trastornos genéticos (síndrome de May Hegglin, síndrome de Fechtner, síndrome de Sebastián y síndrome de Epstein)

Hemodiálisis, hipertensión maligna, válvulas cardíacas protésicas, radiación ionizante, quimioterapia, anafilaxia, falla renal, deficiencia

Otras de vitamina B12 o folatos, enfermedad hepática, hemoglobinuria paroxística nocturna, linfohistiocitosis hemofagocítica, anemia de células falciformes

VIH: virus de inmunodeficiencia primaria; HHV-6: herpesvirus humano 6; EBV: virus de Epstein-Bar.

tos del Registro de Oklahoma de PTT y SHU [15]. La PTT adquirida tiene una edad media de diagnóstico de 41 años, con un rango amplio de 9 a 78 años. Es muy rara en niños (incidencia de 1 por 10 millones/año en menores de 18 años) [16], por lo que se debe considerar la posibilidad de una PTT hereditaria en vez de una adquirida, mientras que en adultos la PTT adquirida es 30 veces más frecuente que la hereditaria [8]. La enfermedad puede afectar a cualquier edad, pero ocurre predominantemente entre los 20 a 50 años, con una relación mujer:hombre de 2 a 1 [17].
Las características demográficas asociadas con un riesgo incrementado de PTT adquirida incluyen el sexo femenino y la raza negra; estas son las mismas características encontradas en pacientes con lupus eritematoso sistémico y con PTT [18].

\section{Fisiopatología}

En 1982, Moake demostró que los pacientes con PTT exhibían multímeros de factor de von Willebrand (FVW) inusualmente grandes, que eran hiperadhesivos a las plaquetas en el 
plasma [19], y en 1985, gracias a la histopatología, pudo establecerse que los microtrombos eran ricos en plaquetas y FVW, y no en fibrinógeno $[20,21]$.

En condiciones fisiológicas, el FVW es liberado por las células endoteliales y queda adherido a la superficie de la célula, donde la metaloproteasa ADAMTS13 actúa a nivel del dominio A2 del FWW, escindiendo los multímeros inusualmente grandes que presentan una gran afinidad por las plaquetas. Luego de esto, el FVW es liberado a la circulación sanguínea con una conformación que no permite su interacción con las plaquetas [19,22].

La PTT es causada por una deficiencia severa o inhibición de la actividad de la proteasa ADAMTS13, que es producida en hígado, riñones y células endoteliales. Esta enzima funciona como una proteasa de clivaje de los multímeros grandes del FVW, segmentándolos a multímeros más pequeños, y previene que los multímeros grandes se acumulen, especialmente en áreas de estrés (arteriolas pequeñas y capilares). Debido a la deficiencia de ADAMTS13, no se produce una adecuada eliminación de los multímeros grandes del FVW, lo cual conduce a una acumulación en la superficie endotelial que causa adherencia de las plaquetas [23]. Esto genera trombos ricos en plaquetas y FVW que producen destrucción de los glóbulos rojos con formación de esquistocitos y trombocitopenia, que facilitan la isquemia orgánica $[12,24]$.

La gran mayoría de casos de PTT (aproximadamente el 95\%) son adquiridos debido a la formación de autoanticuerpos inhibitorios de ADAMTS13 $[5,25]$.

\section{Clínica}

Su presentación es aguda y de curso grave si no se inicia manejo adecuado de manera oportuna. Los pacientes pueden presentar síntomas prodrómicos como fiebre, fatiga, artralgias, mialgias y síntomas gastrointestinales (dolor abdominal, náuseas, vómito y diarrea); también pueden manifestar síntomas asociados a la anemia y trombocitopenia como disnea, ictericia, coluria, petequias u otros sangrados $[26,27]$. Debido a la isquemia producida por los microtrombos, pueden manifestar síntomas neurológicos que pueden ir desde cefalea, hasta convulsiones, accidente cerebrovascular, coma y muerte. No todos los pacientes que se presentan con PTT se ven críticamente enfermos, y en algunos pacientes el diagnóstico de PTT no es considerado hasta que un hemograma completo con extendido de sangre periférica revela los hallazgos de AHMA y trombocitopenia. Típicamente, los pulmones no se comprometen por la injuria isquémica producida por la PTT [9]. Aunque no es usual, se puede observar compromiso cardíaco con infarto agudo de miocardio, arritmias y muerte súbita, además de compromiso gastrointestinal.

\section{Diagnóstico}

Los pacientes con PTT suelen presentar trombocitopenia severa (conteos por debajo de 30.000 plaquetas $/ \mu \mathrm{L}$ ). Además, la PTT es única entre los síndromes de MAT primarios porque produce mínimas anormalidades en la función renal. A pesar de que se han encontrado microtrombos renales en autopsias de pacientes con esta enfermedad, rara vez la creatinina pasa de $2,3 \mathrm{mg} / \mathrm{dL}$ y ocasionalmente los pacientes requieren terapia de reemplazo renal [12]. Con frecuencia el uroanálisis puede 
mostrar hemoglobinuria y proteinuria leves [28-30].

El diagnóstico de la PTT es clínico y la medición de la actividad de ADAMTS13 no debe retrasar el inicio del tratamiento específico para esta entidad, el cual es urgente. Sin embargo, el diagnóstico debe ser confirmado con la medición de ADAMTS13 que tendrá una actividad inferior al 10\%. Se debe asegurar la toma de la muestra para este examen antes del inicio del tratamiento para evitar confusiones. La probabilidad de PTT puede ser calculada por medio del PLASMIC score [31].

En centros donde esté disponible se puede realizar la medición de los anticuerpos contra la enzima ADAMTS13 $y$, adicionalmente, realizar la prueba genética, lo que permite distinguir si la PTT es congénita o adquirida [5].

No se requiere la biopsia de los tejidos para hacer diagnóstico de PTT, pero si es realizada se puede encontrar una maduración normal de las tres líneas celulares con un incremento de la eritropoyesis y trombopoyesis, para compensar el consumo de plaquetas y eritrocitos en la circulación, además de los hallazgos clásicos de la MAT con microtrombos de plaquetas en las arteriolas pequeñas o capilares de los diferentes tejidos [8]. Esta evaluación de la médula ósea se realiza si los hallazgos de la MAT no son claros o si se sospecha un diagnóstico alternativo como una malignidad sistémica [32].

\section{Tratamiento}

El tratamiento de la PTT se realiza con intercambio plasmático diario (1,5 a 2 volemias) hasta normalizar la actividad hemolítica (plaquetas y LDH normales) lo cual permite remover los anticuerpos contra la ADAMTS13 o reemplazar la ADAMTS13 mutada por una enzima normal. Generalmente, cuando se inicia la plasmaféresis por un diagnóstico de PTT también se inician glucocorticoides, ya que la PTT adquirida (autoinmune) es la más frecuente. En casos refractarios se puede utilizar rituximab, ciclofosfamida [33] o caplacizumab; sin embargo, de acuerdo con las guías de la ISTH (del inglés, International Society on Thrombosis and Haemosthasis), se resalta la necesidad de reunir mayor información para el uso de este nuevo medicamento, solo o en combinación [34]. Las guías actuales recomiendan iniciar la plasmaféresis en las primeras 4 a 8 horas de la sospecha clínica de PTT $[35,36]$.

Usualmente se suspende la plasmaféresis luego de que el paciente ha tenido un recuento de plaquetas normal por 2 días. Sin embargo, pueden ocurrir exacerbaciones (disminución del recuento de plaquetas) cuando la plasmaféresis es suspendida, y esto usualmente se da en la primera semana [8].

La PTT es una emergencia médica que puede ser fatal si no se inicia un tratamiento apropiado de forma oportuna. Si no se cuenta con la terapia de intercambio plasmático de forma inmediata, puede ser útil el uso de plasma fresco congelado o concentrado de factor VIII.

\section{Pronóstico}

La mortalidad es hasta del $90 \%$ si no se inicia tratamiento, y del $10 \%$ al $20 \%$ con tratamiento adecuado $[5,37]$. Se ha hablado de biomarcadores que pueden predecir la mortalidad, los cuales incluyen los niveles de anticuerpos contra ADAMTS13, troponina I, tasa de recuperación plaquetaria, hipoproteinemia o hipoalbuminemia y tiempo de tromboplastina parcial activado prolonga- 
do. Recientemente, un artículo reportó la existencia de una correlación entre los niveles de dímero $D$ y el pronóstico de los pacientes con PTT, proponiendo que sea utilizado como biomarcador para la estratificación del riesgo de los pacientes; sin embargo, falta mayor evidencia para generalizar estos hallazgos [38].

\section{Síndrome hemolítico urémico típico}

El SHU mediado por toxina Shiga fue descrito por primera vez por Gasser y colegas en 1955 como una triada clínica de trombocitopenia, anemia y lesión renal aguda [39]. Está clásicamente asociado a la enterocolitis producida por Escherichia coli productora de toxina Shiga (serotipos 0157:H7 y 0104:H4), que produce aproximadamente del $85 \%$ al $95 \%$ de los casos en niños. La otra proporción de casos se da por la toxina Shiga producida por Shigella dysenteriae $[39,40]$.

\section{Epidemiología}

En Estados Unidos y Europa, el organismo responsable del SHU típicamente es la bacteria E. coli O157:H7, O111 u O104:H4 [41-44]. Las especies de Shigella pueden ser más comunes como fuente de toxina Shiga en Asia [8]. Es una enfermedad extremadamente rara que afecta usualmente a niños menores de 5 años con incidencia de 5 a 6 casos por cada 100.000 habitantes, y con mortalidad cercana al 3\%. La distribución es similar en ambos sexos y es una de las principales causas de lesión renal aguda en infantes $[45,46]$. Los pacientes con frecuencia tienen historia de exposición a animales de granja, carne poco cocinada o agua contaminada; típicamente esto ocurre varios días antes del inicio de los síntomas.
En adultos es menos común y se asocia a brotes de disentería, sin embargo, su presentación es más grave con una tasa de mortalidad mayor que en los niños [47].

\section{Fisiopatología}

La infección inicia posterior al consumo de alimentos contaminados con $E$. coli productora de toxina Shiga; esta se une a los enterocitos e induce diarrea y posteriormente es translocada a la circulación llegando al riñón, donde induce la producción de sustancias protrombóticas e inflamatorias, como el FVW y la P-selectina, además de citoquinas que producen activación del complemento y daño multiorgánico. El uso de algunos antibióticos favorece la liberación de toxina Shiga por E. coli y empeoran el cuadro clínico [48].

\section{Clínica}

Los pacientes se presentan con diarrea acuosa que progresa a disentería entre 2 a 12 días después de la exposición a la toxina. El dolor abdominal, las náuseas y el vómito son frecuentes, y a medida que se resuelven estos síntomas aparece el compromiso renal, la anemia y la trombocitopenia [41]. Los pacientes pueden presentar síntomas de la anemia como palidez mucocutánea, ictericia, fatiga y síntomas asociados a la lesión renal como oliguria, edema y disnea. Los síntomas neurológicos se pueden presentar en el $20 \%$ de los niños y en el $50 \%$ de los adultos e incluyen confusión, diplopía, disfagia, parálisis facial, coma, convulsiones y muerte [40]. Otras manifestaciones extrarrenales incluyen compromiso cardiovascular (infarto agudo de miocardio, falla cardíaca congestiva y miocardiopatía dilatada), hemorragia pulmonar, isquemia intestinal y perforación, pancreatitis, diabetes mellitus y colecistitis [49]. 


\section{Diagnóstico}

El diagnóstico es clínico en un paciente con AHMA, trombocitopenia, lesión renal y episodio previo de diarrea. Es importante demostrar la presencia de E. coli productora de toxina Shiga que puede aislarse en coprocultivo, con estudios de FilmArray ${ }^{\circledR}$ gastrointestinal (PCR para E. coli enterohemorrágica) o demostrando la presencia de la toxina en la materia fecal [50]

\section{Tratamiento}

El SHU típico es tratado con terapia de soporte que incluye transfusiones sanguíneas, control de la hipertensión (preferiblemente con nifedipino o nicardipino), hidratación y corrección de trastornos hidroelectrolíticos y hemodiálisis, cuando esté indicada [26]. El beneficio del plasma y del tratamiento anticomplemento es incierto, porque en la mayoría de casos los pacientes mejoran con terapia de soporte. Sin embargo, en los adultos con frecuencia es necesario utilizar estas medidas porque tienen cuadros más agresivos y refractarios al manejo. El intercambio plasmático no tiene muy buena evidencia que lo soporte en niños con SHU típico, y es muy controversial en adultos. A pesar de la falta de evidencia, esta terapia puede ser considerada cuando hay anormalidades neurológicas severas [28].

\section{Pronóstico}

En una revisión sistemática y metaanálisis realizada por Garg y colaboradores con 49 estudios y 3.476 pacientes, se encontró que la muerte o la falla renal en estadio terminal ocurrieron en el $12 \%$ de los pacientes, 4 años después del SHU asociado a diarrea, y $25 \%$ de los que sobrevivieron demostraron secuelas renales a largo plazo. La severidad de la enfermedad aguda, particu- larmente de los síntomas neurológicos y de la necesidad de diálisis, está fuertemente asociada con un pronóstico peor a largo plazo [50].

\section{Microangiopatía trombótica mediada por complemento}

También denominada síndrome hemolítico urémico atípico, es una MAT primaria producida por una regulación defectuosa del complemento, debido a una alteración genética que puede ser disparada por infecciones, trastornos autoinmunes, malignidad sistémica y embarazo, entre otros factores [5]. Se debe evitar usar el término "síndrome hemolítico urémico atípico" para describir a la MAT mediada por complemento porque originalmente fue considerado como un diagnóstico no específico para niños con síndrome hemolítico urémico sin diarrea, y este término no especifica un mecanismo - una terapia apropiada [8]. La MAT mediada por complemento puede ser hereditaria por una mutación genética, o puede ser adquirida por un autoanticuerpo que inhibe la actividad de las proteínas reguladoras.

\section{Epidemiología}

Es un trastorno raro con incidencia de 2 por 1 millón de personas por año. Afecta a todas las edades, pero principalmente a niños y adultos jóvenes. La incidencia de MAT mediada por complemento en niños corresponde aproximadamente al $5 \%$ de todos los $\mathrm{SHU}$ inicialmente clasificados como típicos. La mayoría se deben a mutaciones de los factores del complemento y a los anticuerpos contra las proteínas del complemento, los cuales han sido implicados en la etiología del $6 \%$ al $10 \%$ de los pacientes [51-53]. Ocurre con igual frecuencia en hombres y mujeres 
cuando su inicio se da durante la niñez. En el registro global de SHU atípico de 516 pacientes de todas las edades, el $39 \%$ de los pacientes desarrollaron la enfermedad antes de los 18 años de edad, y aproximadamente el $44 \%$ de los niños experimentó el primer episodio antes de la edad de 2 años. Puede ser esporádico o familiar, con miembros de la familia afectados en un $20 \%$ de los casos infantiles [54].

\section{Fisiopatología}

La cascada del complemento puede causar lisis de las células diana formando poros en la membrana celular. Cualquier célula puede ser una diana para el complemento, incluyendo microorganismos y células del hospedero. La vía alterna protege a las células propias de un ataque inapropiado del complemento y la falla de los mecanismos de control normales para regular esta vía pueden llevar a daño endotelial. Las proteínas reguladoras de la vía alterna del complemento incluyen factores solubles como el factor $\mathrm{H}$, proteína de cofactor de membrana CD46, factor I, factor B, C3 y factores unidos a la membrana como la trombomodulina, entre otros $[55,56]$. La pérdida de función de estos factores reguladores causa una producción excesiva de C3b y C3 convertasa, y aumenta la formación del complejo de ataque de membrana (C5b-C9), con progresión a lesión endotelial, trombosis y daño de órgano blanco [48]. Las células del riñón parecen ser especialmente sensibles a la activación del complemento, lo cual puede explicar el predominio de la lesión renal aguda en la MAT mediada por complemento [8].

\section{Clínica}

El inicio de la MAT mediada por complemento es generalmente súbito.
Puede haber una infección precedente, incluyendo enfermedad diarreica aguda, hasta en el $80 \%$ de los niños y en el $50 \%$ de los adultos. Los síntomas incluyen palidez, malestar general e inapetencia y puede haber edema. La hipertensión arterial y el compromiso renal son frecuentes, y las manifestaciones extrarrenales se pueden observar hasta en el $20 \%$ de los pacientes e incluyen manifestaciones en sistema nervioso central (hallazgo extrarrenal más común), eventos de isquemia cardíaca, hemorragia pulmonar, pancreatitis y sangrado gastrointestinal $[56,57]$.

Esta condición usualmente es precipitada por una causa infecciosa, inmunológica, traumática o medicamentosa en una persona que ya tenía predisposición genética, lo cual ocurre en el $50 \%$ de los niños y en el $70 \%$ de los adultos $[40,58]$.

\section{Diagnóstico}

Se deben descartar otras MAT primarias más frecuentes como PTT y SHU típico antes de considerar la MAT por alteración en la regulación del complemento. Se deben realizar estudios de ADAMTS13, coprocultivo, hemocultivos, toxina Shiga, perfil inmunológico completo, búsqueda de enfermedades infecciosas frecuentes (VIH, hepatotropos y sífilis), y otros, según la clínica. Los niveles bajos de $\mathrm{C} 3$ se encuentran hasta en el $30 \%$ de los casos, por lo que un valor normal no descarta la presencia de este tipo de MAT [59].

Una vez se realiza el tratamiento de soporte y se estabiliza el paciente, es necesario la realización de un estudio genético para establecer pronóstico, realizar asesoría genética a la familia y hacer un plan de tratamiento a largo plazo. Se identifica mutación genética hasta en el $70 \%$ de los casos, pero 
esto no indica que el $30 \%$ restante no tenga la enfermedad, ya que también pueden presentar desregulación del complemento, pero no se identifica la mutación genética.

\section{Tratamiento}

La terapia es de soporte con intercambio plasmático, antihipertensivos, transfusiones, diálisis y manejo de complicaciones mientras se hace el diagnóstico etiológico específico. Cuando se sospecha la MAT mediada por complemento, la terapia anticomplemento (eculizumab) debe ser iniciada tan pronto como sea posible, preferiblemente en las primeras 24 a 48 horas. El objetivo principal es limitar la lesión renal en un estadio irreversible [8].

Antes de iniciar el eculizumab (anticuerpo contra la fracción C5 del complemento), los pacientes deben recibir vacunación contra encapsulados (meningococo, Haemophilus influenzae tipo B y neumococo) y terapia antibiótica profiláctica por 2 semanas posterior al inicio del fármaco [8]. En general, el tratamiento con eculizumab es de por vida para pacientes con mutaciones en el factor $\mathrm{H}$, factor I, C3 y factor $\mathrm{B}$; no obstante, la suspensión del fármaco podría considerarse en pacientes con mutación MCP-CD46 o en quienes no se identificó alguna mutación después de 6 a 12 meses de terapia continua y posterior a 3 meses de recuperación de la función renal [48].

En los niños es razonable iniciar una terapia anti-complemento como primera línea de tratamiento si presentan AHMA, trombocitopenia y falla renal no asociada a diarrea sanguinolenta que sugiera un SHU y sin otros diagnósticos alternativos [8].
El eculizumab es removido por la plasmaféresis y diluido por la infusión de plasma. Es por esto que, si estas terapias son usadas concomitantemente, se requieren dosis adicionales de eculizumab posteriores a cada procedimiento de plasmaféresis [8].

\section{Pronóstico}

En la fase aguda de la enfermedad hay una mortalidad hasta del $30 \%$ y la mayoría de los pacientes (más del 80\%) requieren terapia de reemplazo renal. Sin tratamiento, el $70 \%$ de los pacientes a 1 año de seguimiento fallecen o estarán en diálisis, y si el paciente es trasplantado sin tratamiento, tiene una recurrencia en el injerto renal hasta del $80 \%$ [60].

\section{Abordaje de los pacientes}

La evaluación inicial debe estar enfocada en confirmar la presencia de anemia hemolítica microangiopática no autoinmune, trombocitopenia y excluir trastornos sistémicos que manifiesten estos hallazgos [5]. Una vez que se haya realizado lo anterior, se debe encontrar la MAT primaria que más se ajusta al paciente.

Los estudios iniciales necesarios son el hemograma completo con plaquetas, extendido de sangre periférica, Coombs directo, LDH, bilirrubinas y recuento de reticulocitos. También se requieren pruebas de coagulación que ayudan a diferenciar las MAT primarias de la coagulación intravascular diseminada. De igual forma, se debe evaluar la función renal, enzimas hepáticas, estudios infecciosos $(\mathrm{VIH}$, sífilis, hepatitis $B$ y $C$ ), perfil autoinmune completo y otros exámenes según la clínica del paciente y que ayuden a descartar MAT secundarias [28]. 
La probabilidad de PTT puede ser calculada por medio del PLASMIC score [31]. Si se sospecha PTT se debe tomar la muestra para la medición de la actividad de ADAMTS13, y se debe iniciar manejo con plasmaféresis. Una actividad de ADAMTS13 inferior al 10\% confirma el diagnóstico. Si se encuentra infección por E. coli enterohemorrágica en coprocultivo o se encuentra la toxina Shiga, se hace diagnóstico de SHU típico y se debe dar terapia de soporte. Si se encuentra causa secundaria asociada a la MAT, se hace el tratamiento de la misma. Si se descarta todo lo anterior o el paciente no responde al manejo instaurado para otro tipo de MAT, se debe considerar MAT media- da por complemento e iniciar manejo con eculizumab. Debido a que el eculizumab puede prevenir la enfermedad renal en estadio terminal y revertir la injuria renal aguda, su uso empírico es apropiado en pacientes con falla renal aguda con progresión rápida [8].

En la figura 1 se resume el abordaje para el diagnóstico diferencial y tratamiento de las MAT.

\section{Conclusión}

Aunque las MAT, son patologías infrecuentes, son enfermedades que sin un diagnóstico y tratamiento oportuno

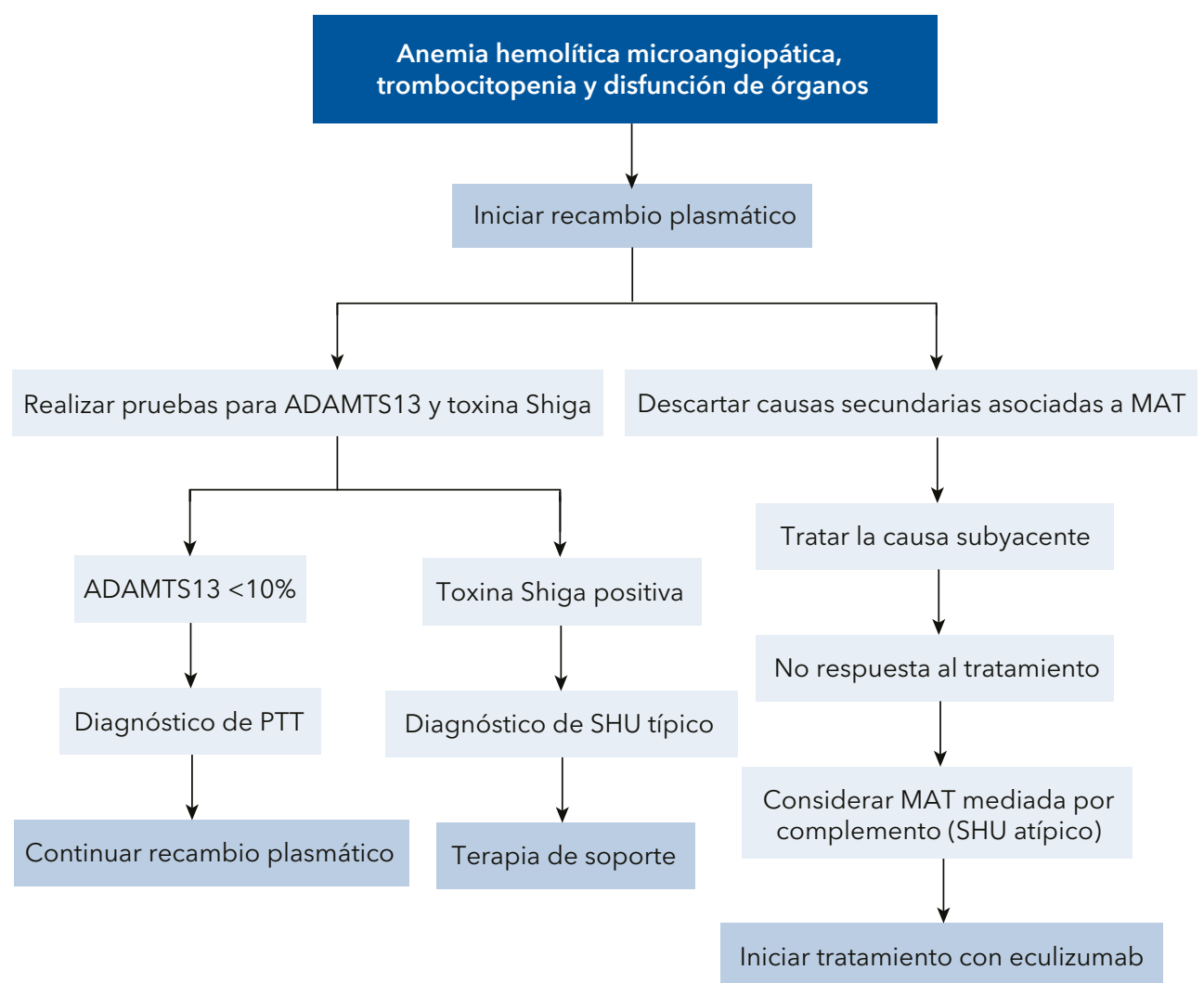

Figura 1. Algoritmo para el diagnóstico diferencial y manejo de las MAT. 
pueden generar una alta morbilidad y mortalidad, y es por esto que, ante la sospecha clínica de una MAT, se debe instaurar tratamiento inmediato con plasmaféresis, sin dejar de lado la búsqueda de la causa de este síndrome. Uno de los problemas más importantes en estos pacientes es la demora desde que el paciente recibe la primera atención médica hasta que se orienta el diagnóstico de MAT, lo que conduce a retraso del tratamiento y a complicaciones irreversibles. Es por esto que, ante la presencia de anemia microangiopática y trombocitopenia grave, está indicado iniciar lo antes posible el recambio plasmático, lo que mejora la respuesta y pronóstico de la enfermedad $[2,37,61-63]$.

\section{Referencias}

1. Moschcowitz E. An acute febrile pleiochromic anemia with hyaline thrombosis of the terminal arterioles and capillaries; an undescribed disease. Am J Med 1952;13:567-569. https://doi. org/10.1016/0002-9343(52)90022-3.

2. Contreras E, De la Rubia J, del Río-Garma J, Díaz-Ricart M, García-Gala JM, Lozano M. Guía diagnóstica y terapéutica de las microangiopatías trombóticas del Grupo Español de Aféresis. Med Clin 2015;144:e1-e13. https:// doi.org/10.1016/j.medcli.2014.09.013.

3. Arnold DM, Patriquin CJ, Nazy I. Thrombotic microangiopathies: a general approach to diagnosis and management. CMAJ 2017;189:E153E159. https://doi.org/10.1503/cmaj.160142.

4. Radhakrishnan J. Anticomplement therapies in "secondary thrombotic microangiopathies": ready for prime time? Kidney Int 2019;96:833-835. https://doi.org/10.1016/j.kint.2019.08.005.

5. George JN, Nester CM. Syndromes of thrombotic microangiopathy. N Engl J Med 2014;371:654-666. https://doi.org/10.1056/NEJMra1312353.

6. Moake JL. Thrombotic microangiopathies. N Engl J Med 2002;347:589-600. https://doi. org/10.1056/NEJMra020528.
7. Romero S, Sempere A, Gómez-Seguí I, Román E, Moret A, Jannone R, et al. Guía práctica de tratamiento urgente de la microangiopatía trombótica. Med Clin 2018;151:123 e121-123.e129. https://doi.org/https://doi. org/10.1016/j.medcli.2018.01.013.

8. George JN, Nester CM. Approach to the patient with suspected TTP, HUS, or other thrombotic microangiopathy (TMA). Massachusetts, Estados Unidos: Wolters Kluwer Health; 2019 Acceso 20 de agosto de 2020. Disponible en https://www.uptodate.com/contents/approachto-the-patient-with-suspected-ttp-hus-or-otherthrombotic-microangiopathy-tma\#H16673902.

9. Nokes T, George JN, Vesely SK, Awab A. Pulmonary involvement in patients with thrombotic thrombocytopenic purpura. Eur J Haematol 2014;92:156-163. https://doi. org/10.1111/ejh.12222.

10. Blasco-Pelicano M, Guillé E, Quintana L, Garcia-Herrera A, Piñeiro G, Poch E, et al. Thrombotic microangiopathies assessment: mind the complement. Clin Kidney J 2020;2020:sfaa195. https://doi.org/10.1093/ckj/sfaa195.

11. Eskazan AE, Salihoglu A. Treatment and outcome of primary and secondary thrombotic microangiopathies. Am J Nephrol 2015;41:427428. https://doi.org/10.1159/000437002.

12. Kremer Hovinga JA, Coppo $P$, Lämmle B, Moake JL, Miyata T, Vanhoorelbeke K. Thrombotic thrombocytopenic purpura. Nat Rev Dis Primers 2017;3:17020. https://doi. org/10.1038/nrdp.2017.20.

13. Joly BS, Coppo P, Veyradier A. An update on pathogenesis and diagnosis of thrombotic thrombocytopenic purpura. Expert Rev Hematol 2019;12:383-395. https://doi.org/10.1080/ 17474086.2019.1611423.

14. Shatzel JJ, Taylor JA. Syndromes of thrombotic microangiopathy. Med Clin North Am 2017;101:395-415. https://doi.org/10.1016/j. mcna.2016.09.010.

15. George JN. Thrombotic microangiopathy (TMA). Platelets on the Web. Oklahoma, USA: University of Oklahoma Health Sciences Center: 2007. Acceso 5 de agosto de 2020. Disponible en https://www.ouhsc.edu/platelets/TMA.htm.

16. Reese JA, Muthurajah DS, Kremer Hovinga JA, Vesely SK, Terrell DR, George JN. Chil- 
dren and adults with thrombotic thrombocytopenic purpura associated with severe, acquired Adamts13 deficiency: comparison of incidence, demographic and clinical features. Pediatr Blood Cancer 2013;60:1676-1682. https://doi. org/10.1002/pbc.24612.

17. Terrell DR, Williams LA, Vesely SK, Lämmle B, Hovinga JA, George JN. The incidence of thrombotic thrombocytopenic purpura-hemolytic uremic syndrome: all patients, idiopathic patients, and patients with severe ADAMTS-13 deficiency. J Thromb Haemost 2005;3:1432-1436. https:// doi.org/10.1111/j.1538-7836.2005.01436.x.

18. Terrell DR, Vesely SK, Kremer Hovinga JA, Lämmle B, George JN. Different disparities of gender and race among the thrombotic thrombocytopenic purpura and hemolytic-uremic syndromes. Am J Hematol 2010;85:844-847. https://doi.org/10.1002/ajh.21833.

19. Moake JL, Rudy CK, Troll JH, Weinstein MJ, Colannino NM, Azocar J, et al. Unusually large plasma factor VIII:von Willebrand factor multimers in chronic relapsing thrombotic thrombocytopenic purpura. N Engl J Med 1982;307:1432-1435. https://doi.org/10.1056/ nejm198212023072306.

20. Asada Y, Sumiyoshi A, Hayashi T, Suzumiya J, Kaketani K. Immunohistochemistry of vascular lesion in thrombotic thrombocytopenic purpura, with special reference to factor VIII related antigen. Thromb Res 1985;38:469-479. https:// doi.org/10.1016/0049-3848(85)90180-x.

21. Vacca VM, Jr. Acquired autoimmune thrombotic thrombocytopenic purpura. Nursing 2019;49:22-29. https://doi.org/10.1097/01. NURSE.0000549721.69197.4d.

22. Moake JL, Turner NA, Stathopoulos NA, Nolasco LH, Hellums JD. Involvement of large plasma von Willebrand factor (vWF) multimers and unusually large vWF forms derived from endothelial cells in shear stress-induced platelet aggregation. J Clin Invest 1986;78:14561461. https://doi.org/10.1172/jci112736.

23. Zander CB, Cao W, Zheng $\mathbf{X L}$. ADAMTS13 and von Willebrand factor interactions. Curr Opin Hematol 2015;22:452-459. https://doi. org/10.1097/moh.0000000000000169.

24. Furlan $M$, Robles $R$, Galbusera $M$, Remuzzi G, Kyrle PA, Brenner B, et al. von Willebrand factor- cleaving protease in thrombotic thrombocytopenic purpura and the hemolytic-uremic syndrome. N Engl J Med 1998;339:1578-1584. https:// doi.org/10.1056/nejm199811263392202.

25. Blombery P, Scully M. Management of thrombotic thrombocytopenic purpura: current perspectives. J Blood Med 2014;5:15-23. https:// doi.org/10.2147/jbm.S46458.

26. Kappler S, Ronan-Bentle S, Graham A. Thrombotic microangiopathies (TTP, HUS, HELLP). Hematol Oncol Clin North Am 2017;31:1081-1103. https://doi.org/10.1016/j. hoc.2017.08.010.

27. Griffin D, Al-Nouri ZL, Muthurajah D, Ross JR, Ballard RB, Terrell DR, et al. First symptoms in patients with thrombotic thrombocytopenic purpura: what are they and when do they occur? Transfusion 2013;53:235-237. https://doi. org/10.1111/j.1537-2995.2012.03934.x.

28. George JN. How I treat patients with thrombotic thrombocytopenic purpura: 2010. Blood 2010;116:4060-4069. https://doi.org/10.1182/ blood-2010-07-271445.

29. Vesely SK, George JN, Lämmle B, Studt JD, Alberio L, El-Harake MA, et al. ADAMTS13 activity in thrombotic thrombocytopenic purpura-hemolytic uremic syndrome: relation to presenting features and clinical outcomes in a prospective cohort of 142 patients. Blood 2003;102:60-68. https://doi. org/10.1182/blood-2003-01-0193.

30. Brocklebank V, Wood KM, Kavanagh D. Thrombotic microangiopathy and the kidney. Clin J Am Soc Nephrol 2018;13:300-317. https://doi.org/10.2215/cjn.00620117.

31. Bendapudi PK, Hurwitz S, Fry A, Marques MB, Waldo SW, Li A, et al. Derivation and external validation of the PLASMIC score for rapid assessment of adults with thrombotic microangiopathies: a cohort study. Lancet Haematol 2017;4:e157-e164. https://doi.org/10.1016/ s2352-3026(17)30026-1.

32. George JN. Systemic malignancies as a cause of unexpected microangiopathic hemolytic anemia and thrombocytopenia. Oncology 2011;25:908-914.

33. Furlan $M$, Robles $R$, Morselli $B$, Sandoz $\mathbf{P}$, Lämmle B. Recovery and half-life of von Willebrand factor-cleaving protease after plasma the- 
rapy in patients with thrombotic thrombocytopenic purpura. Thromb Haemost 1999;81:8-13.

34. Zheng $X L$, Vesely SK, Cataland SR, Coppo P, Geldziler B, lorio A, et al. ISTH guidelines for treatment of thrombotic thrombocytopenic purpura. J Thromb Haemost 2020;18:24962502. https://doi.org/10.1111/jth.15010.

35. Scully M, Hunt BJ, Benjamin S, Liesner R, Rose P, Peyvandi F, et al. Guidelines on the diagnosis and management of thrombotic thrombocytopenic purpura and other thrombotic microangiopathies. $\mathrm{Br} J$ Haematol 2012;158:323-335. https://doi.org/10.1111/ j.1365-2141.2012.09167.x.

36. Schwartz J, Padmanabhan A, Aqui N, Balogun RA, Connelly-Smith L, Delaney M, et al. Guidelines on the use of therapeutic apheresis in clinical practice-evidence-based approach from the Writing Committee of the American Society for Apheresis: The seventh special issue. J Clin Apher 2016;31:149-162. https://doi. org/10.1002/jca.21470.

37. Scully M, Cataland S, Coppo P, de la Rubia J, Friedman KD, Kremer Hovinga J, et al. Consensus on the standardization of terminology in thrombotic thrombocytopenic purpura and related thrombotic microangiopathies. J Thromb Haemost 2017;15:312-322. https:// doi.org/10.1111/jth.13571.

38. Wang HX, Han B, Zhao YY, Kou L, Guo LL, Sun TW, et al. Serum D-dimer as a potential new biomarker for prognosis in patients with thrombotic thrombocytopenic purpura. Medicine (Baltimore) 2020;99:e19563. https://doi. org/10.1097/md.0000000000019563.

39. Gasser C, Gautier E, Steck A, Siebenmann RE, Oechslin R. [Hemolytic-uremic syndrome: bilateral necrosis of the renal cortex in acute acquired hemolytic anemia]. Schweiz Med Wochenschr 1955;85:905-909.

40. Fakhouri F, Zuber J, Frémeaux-Bacchi V, Loirat C. Haemolytic uraemic syndrome. Lancet 2017;390:681-696. https://doi.org/10.1016/s01406736(17)30062-4.

41. Tarr PI, Gordon CA, Chandler WL. Shiga-toxinproducing Escherichia coli and haemolytic uraemic syndrome. Lancet 2005;365:1073-1086. https://doi.org/10.1016/s0140-6736(05)711442.
42. Witham PK, Yamashiro $C T$, Livak $\mathrm{KJ}$, Batt CA. A PCR-based assay for the detection of Escherichia coli Shiga-like toxin genes in ground beef. Appl Environ Microbiol 1996;62:13471353. https://doi.org/10.1128/aem.62.4.13471353.1996.

43. Bell BP, Goldoft $M$, Griffin PM, Davis MA, Gordon DC, Tarr PI, et al. A multistate outbreak of Escherichia coli O157:H7-associated bloody diarrhea and hemolytic uremic syndrome from hamburgers. The Washington experience. Jama 1994;272:1349-1353.

44. Buchholz U, Bernard $H$, Werber D, Böhmer MM, Remschmidt C, Wilking $H$, et al. German outbreak of Escherichia coli 0104:H4 associated with sprouts. N Engl J Med 2011;365:1763-1770. https://doi.org/10.1056/ NEJMoa1106482.

45. Noris M, Remuzzi G. Hemolytic uremic syndrome. J Am Soc Nephrol 2005;16:1035-1050. https://doi.org/10.1681/asn.2004100861.

46. Cody EM, Dixon BP. Hemolytic uremic syndrome. Pediatr Clin North Am 2019;66:235-246. https://doi.org/10.1016/j.pcl.2018.09.011.

47. Karpac CA, Li $X$, Terrell DR, Kremer Hovinga JA, Lämmle B, Vesely SK, et al. Sporadic bloody diarrhoea-associated thrombotic thrombocytopenic purpura-haemolytic uraemic syndrome: an adult and paediatric comparison. Br J Haematol 2008;141:696-707. https://doi. org/10.1111/j.1365-2141.2008.07116.x.

48. Manrique-Caballero $C L$, Peerapornratana S, Formeck C, Del Rio-Pertuz G, Gomez Danies $\mathbf{H}$, Kellum JA. Typical and atypical hemolytic uremic syndrome in the critically ill. Crit Care Clin 2020;36:333-356. https://doi. org/10.1016/j.ccc.2019.11.004.

49. Spinale JM, Ruebner RL, Copelovitch L, Kaplan BS. Long-term outcomes of Shiga toxin hemolytic uremic syndrome. Pediatr Nephrol 2013;28:2097-2105. https://doi.org/10.1007/ s00467-012-2383-6.

50. Garg AX, Suri RS, Barrowman N, Rehman F, Matsell D, Rosas-Arellano MP, et al. Longterm renal prognosis of diarrhea-associated hemolytic uremic syndrome: a systematic review, meta-analysis, and meta-regression. Jama 2003;290:1360-1370. https://doi.org/10.1001/ jama.290.10.1360. 
51. Taylor CM, Machin S, Wigmore SJ, Goodship TH. Clinical practice guidelines for the management of atypical haemolytic uraemic syndrome in the United Kingdom. Br J Haematol 2010;148:37-47. https://doi.org/10.1111/ j.1365-2141.2009.07916.x.

52. Geerdink LM, Westra D, van Wijk JA, Dorresteijn EM, Lilien MR, Davin JC, et al. Atypical hemolytic uremic syndrome in children: complement mutations and clinical characteristics. Pediatr Nephrol 2012;27:1283-1291. https:// doi.org/10.1007/s00467-012-2131-y.

53. Hofer J, Janecke AR, Zimmerhackl LB, RiedI M, Rosales A, Giner T, et al. Complement factor H-related protein 1 deficiency and factor $\mathrm{H}$ antibodies in pediatric patients with atypical hemolytic uremic syndrome. Clin J Am Soc Nephrol 2013;8:407-415. https://doi. org/10.2215/cjn.01260212.

54. Licht C, Ardissino G, Ariceta G, Cohen D, Cole JA, Gasteyger $\mathbf{C}$, et al. The global aHUS registry: methodology and initial patient characteristics. BMC Nephrol 2015;16:207. https://doi. org/10.1186/s12882-015-0195-1.

55. Makou E, Herbert AP, Barlow PN. Functional anatomy of complement factor $\mathrm{H}$. Biochemistry 2013;52:3949-3962. https://doi.org/10.1021/ bi4003452.

56. Noris M, Caprioli J, Bresin E, Mossali C, Pianetti G, Gamba S, et al. Relative role of genetic complement abnormalities in sporadic and familial aHUS and their impact on clinical phenotype. Clin J Am Soc Nephrol 2010;5:18441859. https://doi.org/10.2215/cjn.02210310.

57. Sellier-Leclerc AL, Fremeaux-Bacchi V, Dragon-Durey MA, Macher MA, Niaudet P, Guest
G, et al. Differential impact of complement mutations on clinical characteristics in atypical hemolytic uremic syndrome. J Am Soc Nephrol 2007;18:2392-2400. https://doi.org/10.1681/ asn.2006080811.

58. Goodship TH, Cook HT, Fakhouri F, Fervenza FC, Frémeaux-Bacchi V, Kavanagh D, et al. Atypical hemolytic uremic syndrome and C3 glomerulopathy: conclusions from a "Kidney disease: Improving global outcomes" (KDIGO) Controversies Conference. Kidney Int 2017;91:539-551. https://doi.org/10.1016/j.kint.2016.10.005.

59. Cataland SR, Holers VM, Geyer S, Yang S, Wu HM. Biomarkers of terminal complement activation confirm the diagnosis of aHUS and differentiate aHUS from TTP. Blood 2014;123:3733-3738. https://doi.org/10.1182/ blood-2013-12-547067.

60. Noris M, Remuzzi G. Atypical hemolytic-uremic syndrome. N Engl J Med 2009;361:1676-1687. https://doi.org/10.1056/NEJMra0902814.

61. Masias C, Vasu S, Cataland SR. None of the above: thrombotic microangiopathy beyond TTP and HUS. Blood 2017;129:2857-2863. https:// doi.org/10.1182/blood-2016-11-743104.

62. Gordon CE, Chitalia VC, Sloan JM, Salant DJ, Coleman DL, Quillen K, et al. Thrombotic microangiopathy: A multidisciplinary team approach. Am J Kidney Dis 2017;70:715-721. https://doi.org/10.1053/j.ajkd.2017.05.017.

63. Bittencourt CE, Ha JP, Maitta RW. Re-examination of 30-day survival and relapse rates in patients with thrombotic thrombocytopenic purpura-hemolytic uremic syndrome. PLoS One 2015;10:e0127744. https://doi.org/10.1371/journal.pone. 0127744 . 